\title{
Erratum to: Genomic analysis in the clinic: benefits and challenges for health care professionals and patients in Brazil
}

\author{
Patrícia Ashton-Prolla ${ }^{1,2,4}$. José Roberto Goldim ${ }^{2,3,4}$ - Filippo Pinto e Vairo ${ }^{1}$. \\ Ursula da Silveira Matte ${ }^{2,4}$ • Jorge Sequeiros ${ }^{5,6,7}$
}

Published online: 29 July 2015

(C) Springer-Verlag Berlin Heidelberg 2015

Erratum to: J Community Genet

DOI 10.1007/s12687-015-0238-0

The original publication of this article unfortunately captured one of the authors' name incorrectly, resulting to incorrect name abbreviation in some indexes. Instead of E VAIRO FP, it should have been VAIRO FP.

The online version of the original article can be found at http://dx.doi.org/ 10.1007/s12687-015-0238-0.

Patrícia Ashton-Prolla

pprolla@gmail.com

1 Serviço de Genetica Medica, Hospital de Clínicas de Porto Alegre, Porto Alegre, RS, Brazil

2 Universidade Federal do Rio Grande do Sul, Porto Alegre, RS, Brazil

3 Serviço de Bioética, Hospital de Clinicas de Porto Alegre, Porto Alegre, RS, Brazil

4 Centro de Pesquisa Experimental, Hospital de Clinicas de Porto Alegre, Porto Alegre, RS, Brazil

5 i3S- Instituto de Investigação e Inovação em Saúde, Universidade do Porto, Porto, Portugal

6 IBMC - Institute for Molecular and Cell Biology, Universidade do Porto, Porto, Portugal

7 ICBAS, Universidade do Porto, Porto, Portugal 